\title{
Fatigability Assessment Using the Fatigue Index Kliniken Schmieder (FKS) Is Not Compromised by Depression
}

\author{
Christian Dettmers ${ }^{1,2}$, Markus Riegger ${ }^{3}$, Oliver Müller ${ }^{4}$, Manfred Vieten ${ }^{3}$ \\ ${ }^{1}$ Kliniken Schmieder Konstanz, Konstanz, Germany \\ ${ }^{2}$ Lurija Institut, Kliniken Schmieder Allensbach, Konstanz, Germany \\ ${ }^{3}$ Sportwissenschaften, Universität Konstanz, Konstanz, Germany \\ ${ }^{4}$ Zentrum für Psychiatrie Reichenau, Konstanz, Germany \\ Email: *c.dettmers@kliniken-schmieder.de
}

How to cite this paper: Dettmers, C., Riegger, M., Müller, O. and Vieten, M. (2016) Fatigability Assessment Using the Fatigue Index Kliniken Schmieder (FKS) Is Not Compromised by Depression. Health, 8, 1485-1494.

http://dx.doi.org/10.4236/health.2016.814147

Received: October 10, 2016

Accepted: November 11, 2016

Published: November 14, 2016

Copyright $\odot 2016$ by authors and Scientific Research Publishing Inc. This work is licensed under the Creative Commons Attribution International License (CC BY 4.0).

http://creativecommons.org/licenses/by/4.0/

\section{(c) (i) Open Access}

\begin{abstract}
Background: Attractor-based kinematic gait analysis using the Fatigue Index Kliniken Schmieder (FKS), have been suggested as a sensitive tool to determine motor fatigability in persons with Multiple Sclerosis (pwMS). Hypothesis: Depression does not affect the FKS to a similar degree as in pwMS. Methods: We recruited 32 patients with major depression. Data were collected with two accelerometers attached to both ankles. Data were recorded for one minute at the beginning and at the end of the treadmill test. Attractor attributes were used to analyze the data. Results: The mean Fatigue Index Kliniken Schmieder (FKS) was 2.1. The change of the attractor $(\delta M)$ was 3.7 and the change of variability $(\delta D)$ was 0.6 . Mean values were clearly below values of pwMS with fatigability from a previous publication. However, the individual level values from six individuals-five of them showed pain related comorbidity besides depression-fell within the lower pathological range. There was no correlation between Hamilton depression scale and the attractor attributes ( $\delta M$ and $\delta D$ ). Discussion: Depression does not affect gait as motor fatigability does in pwMS. Results from subjects with pain during the treadmill test show a moderately increased variability and moderately altered attractors.
\end{abstract}

\section{Keywords}

Accelerometer, Assessment, Attractor, Depression, Fatigue, Gait Analysis, Fatigability, Multiple Sclerosis 


\section{Introduction}

Fatigue is a prominent and frequent symptom in persons with MS (pwMS). It affects up to ninety percent of patients [1] [2]. Fatigue is often experienced as the most disabling and limiting symptom, and greatly contributes to the degradation of general wellbeing, quality of life, and social participation [3] [4]. Moreover, the impact of fatigue at the workplace can be severe and frequently triggers early retirement, even in the early phases of the disease [5]. Despite its high socio-economic impact and its relevance for quality of life diagnostic criteria of fatigue are not well defined.

There is an overlap between cognitive deficit, depression and fatigue [6] especially with respect to cognitive fatigue. It is difficult to unravel those components. It is also quite problematic to diagnose fatigue in the presence of serious depression or sleep disturbances [7]. A major problem in the clinical and scientific field of fatigue is the unclear terminology and contrasting or at least not unified definitions. The discrimination between fatigue and fatigability has been a helpful differentiation [8]: Fatigue represents the subjective sensation of the patient. It is often assessed by a variety of well-established patient questionnaires [6]. Fatigability means change of performance. Cognitive fatigability has been measured by alertness measurements or increasing reaction time in the course of the day [9] or after a standardized cognitive load [10]. Motor fatigability may be easier to diagnose than cognitive fatigability. Clinicians should suspect motor fatigability in a particular pwMS when the reduction of maximal walking distance cannot be explained by the degree of spasticity, paresis or ataxia [11]. During the last years we have developed a method to quantify and objectively assess changes of gait pattern during walking exercise. Kinematic gait analysis with the use of an attractor model is sensitive to detect changes in gait and particularly to identify increasing variability during continued walking [11]. Specificity of increased variability in a gait pattern is not yet established. Since an overlap between depression and fatigue is a common finding [6] the intention of our study was to corroborate that depression does not produce similar results in gait analysis. The following investigation addressed this hypothesis directly: to test whether the Fatigue Index Kliniken Schmieder (FKS) [12] is affected by depression or whether patients with major depression might have a false positive or pathological FKS.

\section{Materials and Methods}

Patients. In a nearby psychiatric hospital (Zentrum Psychiatrie Reichenau) patients were identified who had major depression and who were participating in walking group therapy. There were no further inclusion criteria specified. Exclusion criteria were the very acute stage of depression with suicidal intentions, general unstable conditions or serious heart disease. Patients were informed that the intention of the study was to test their walking capacity and to compare the values with those from a neurological patient group. Thirty-two patients (18 females \& 14 males) were included in the study. Mean time of hospitalization was $89.8 \pm 35.0$ days. Besides antidepressive medication, psychological individual and group interventions, creative therapy and social consultation 
participation in a walking group was part of their therapy. Patients were $50.0 \pm 7.1$ years old. Hamilton depression scale (HDS; seven-item short form [13] was $10.8 \pm 3.8$ (indicating moderate depressive symptoms). Patients received written information concerning the performance of the study. All signed informed consent. The study was approved by the ethical committee of the University of Konstanz and was in agreement with the declaration of Helsinki (Table 1).

Treadmill Test: At the beginning of the walking test the patient's comfortable walking speed was determined individually. The test was performed with the comfortable walking speed plus $10 \%$ and kept constant during the test. Maximal possible speed of the treadmill was $5 \mathrm{~km} / \mathrm{h}$. Patients were asked at regular intervals to score their effort on the Borg Scale ranging from 6 (no effort at all) to 20 (maximal exhaustion you can imagine). Data was collected at two different one-minute intervals: at the beginning of the walking test and once the patient reached 17 ("very hard") on the Borg scale or after a maximal walking time of 1 hour when they did not reach an equivalent effort of "very hard".

Data acquisition: Gait data was recorded using two accelerometers (RehaWatch Motion Sensors, Hasomed GmbH, Magdeburg, Germany; sampling rate $600 \mathrm{~Hz}$ ) attached to both lateral ankles. Data was transferred to a laptop through Bluetooth.

Data analysis: We applied a new method for quantifying movement pattern changes and changes of movement variability using attractor attributes of the acceleration of the feet [12]. Traditional gait analysis uses parameters as stride length, stride frequency, sway, circumduction etc. to analyze walking. These parameters, however, are not sensitive nor stable enough to detect subtle movement changes in individuals. A limit cycle attractor, as used in this and in our previous studies, proves to be very stable for an individual. Such an attractor is a kind of "averaged" movement cycle, to which all movement is attracted. It changes only if system conditions change. The limit cycle attractors-in our case the averaged movement in acceleration space-were approximated and three measures calculated: $\delta M$ represents the velocity-normalized mean distance between two attractors quantifying the dissimilarities between two movement patterns. $\delta D$ denotes the change in movement variability between the beginning and the end of the walk. Here variability is defined as the mean distance between state vector and the attractor. $\delta F$ is the product of $\delta M$ and $\delta D$ and represents an index of the change which we termed the FKS [11].

$$
\delta F=\delta M \cdot \delta D
$$

Table 1. Patients' characteristics.

\begin{tabular}{cc}
\hline Participants (n) & 32 \\
\hline Female (n) & 18 \\
Age (years) & $50 \pm 7.1$ \\
Hamilton Depression Scale & $10.8 \pm 3.8$ \\
Duration of Hospitalization (days) & $90 \pm 35$ \\
\hline
\end{tabular}


We used three-dimensional acceleration measurements $\left(\boldsymbol{a}_{s}(t)\right)$ of two markers ( $s=l, r$ with $l=$ left foot; $r=$ right foot). The start and the end of a loop were defined as the passing of $\boldsymbol{a}_{s}(t)$ through a well-defined 2D plain. The attractor parameter

$$
\begin{aligned}
\boldsymbol{A}_{\mathrm{s}, \mathrm{C}}\left(\tau_{j}\right) & =\frac{1}{n} \sum_{i=1}^{n} \boldsymbol{a}_{\mathrm{s}, \mathrm{C}}\left(i \cdot \tau_{j}\right)+\frac{1}{n} \sum_{i=1}^{n} \boldsymbol{b}_{\mathrm{s}, \mathrm{C}}\left(t=i \cdot \tau_{j}\right) \\
& \approx \frac{1}{n} \sum_{i=1}^{n} \boldsymbol{a}_{\mathrm{s}, \mathrm{C}}\left(i \cdot \tau_{j}\right)
\end{aligned}
$$

was approximated as the acceleration at time $\tau$ (ordering parameter of a complete cycle) averaged over $n$ loops. It follows

$$
\delta M=\frac{1}{v} \sqrt{\left\langle\left(\boldsymbol{A}_{r, B}-\boldsymbol{A}_{r, E}\right)^{2}\right\rangle+\left\langle\left(\boldsymbol{A}_{l, B}-\boldsymbol{A}_{l, E}\right)^{2}\right\rangle}
$$

and

$$
\delta D=\sqrt{\left\langle\left(D_{r, B}-D_{r, E}\right)^{2}\right\rangle+\left\langle\left(D_{l, B}-D_{l, E}\right)^{2}\right\rangle}
$$

with

$$
D_{\mathrm{a}, \mathrm{C}}\left(\tau_{j}\right)=\sqrt{\frac{1}{n-1} \sum_{i=1}^{n}\left[\boldsymbol{A}_{\mathrm{s}, \mathrm{C}}\left(\tau_{j}\right)-\boldsymbol{a}_{\mathrm{s}, \mathrm{C}}\left(i \cdot \tau_{j}\right)\right]^{2}}
$$

and $C=B$ or $E$ (measurement at beginning $(B)$ or end $(E)$ ); $v$ being the walking velocity.

Statistics: Statistical calculations were performed using StatFree Version 8.1.0.2 (Vieten Dynamics, University of Konstanz, Germany) and Microsoft Office Excel 2013. Simple linear regression curves were used for correlation between HDS, $\delta M, \delta D$ and $\delta F$.

\section{Results}

Performance: Most of the patients managed to walk on the treadmill for one hour. The exceptions were two patients, who stopped after 13 and 35 minutes respectively. Average speed was $4.5 \pm 0.7 \mathrm{~km} / \mathrm{h}$. At the end of the walking period patients reached $12.7 \pm$ 2.4 on the Borg scale ("somewhat hard").

Group results of gait analysis: Mean results of the attractor attributes fell within the normal range of non-fatiguing subjects as defined previously [11]. Mean FKS was 2.1 (normal range 0 to 4 ), mean change of the attractor $(\delta M)$ was 3.7 (normal range 1 to 3.6) and mean change of the variability $\delta D$ was 0.6 (normal range 0.9 to 4.1 ). While the attractor changed slightly during the exercise, variability was very stable (Figure 1). Figure 1 illustrates that patients with depression build a different cluster than MS patients with fatigability (published previously, [11]).

Individual results: There were six patients, whose FKS lay outside the normal range within the very low spectrum of the pathological values. Besides the main diagnosis of depression five of them had the following side diagnoses: M. Behcet, lumbar radiculopathy, cervical spondylosis, arthrosis and stroke. These five patients reported pain at the end of the walking. There were no high FKS values as in pwMS (compare Figure 2). 


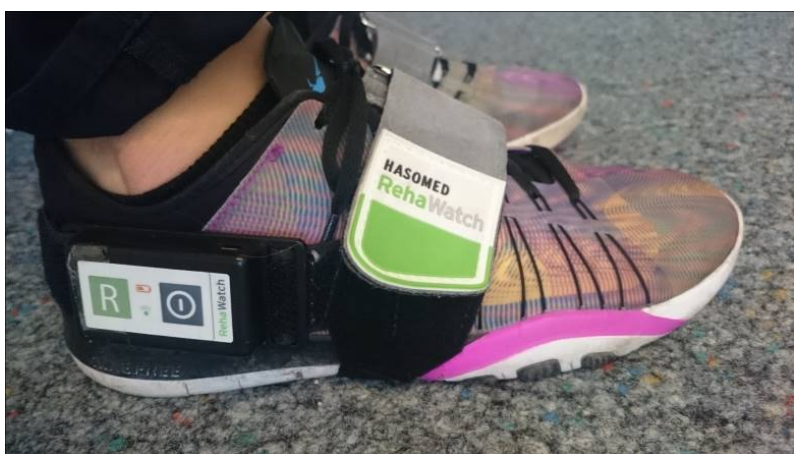

Figure 1. RehaWatch sensors (Hasomed $\mathrm{GmbH}$ ).

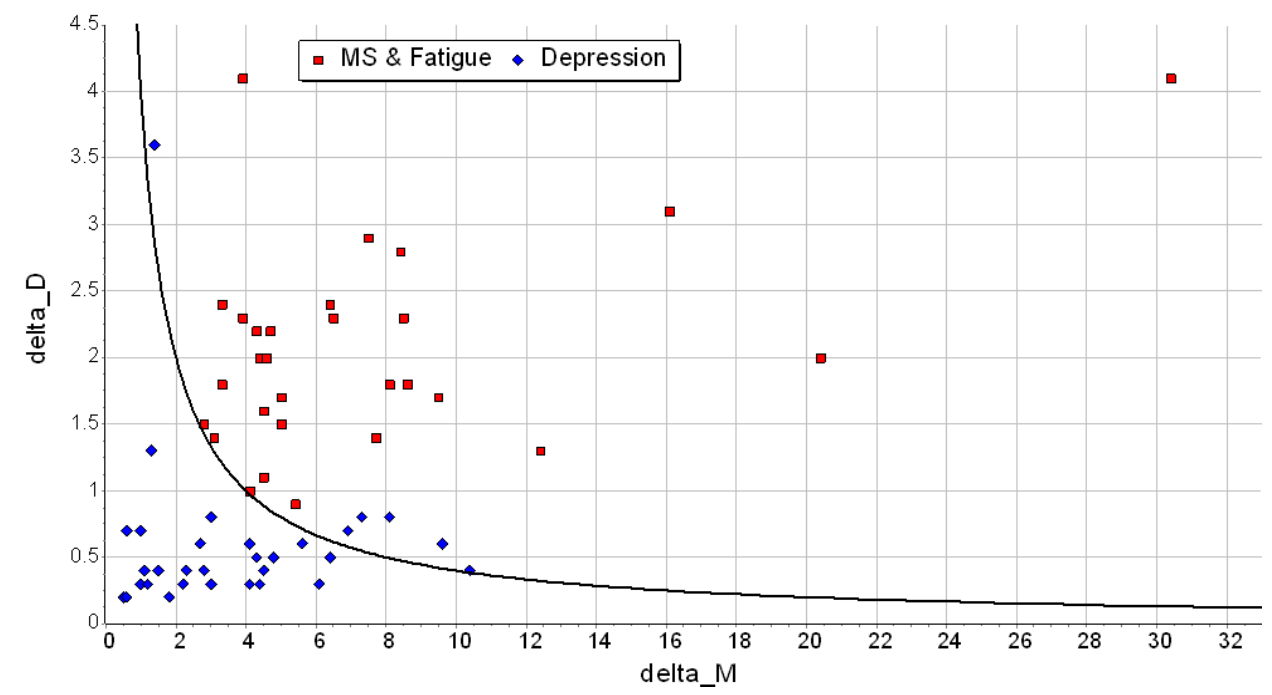

Figure 2. Fatigue Index Kliniken Schmieder of 32 depressive patients (in blue) compared to 29 patients with MS and motor fatigability (in red). Values from pwMS with motor fatigability are taken from [11] (Figure 4 of [11]). The Fatigue Index Kliniken Schmieder is the product of $\delta M$ and $\delta D(\delta F=\delta M \cdot \delta D)$. The figure demonstrates that the values from depressive patients build a different cluster than pwMS and motor fatigability. The black line marks the border between fatigability (upper right area) and non-fatigability (lower left area).

Remarkably, the two patients who did not manage to walk for one hour did not show abnormalities in their attractor attributes.

Correlation analysis between HDS and FKS: There was no significant correlation between the attractor attributes $(\delta M$ and $\delta D)$ and the Hamilton Depression Scale (Figure 3).

\section{Discussion}

This gait analysis of patients with major depression demonstrates that depression does not cause changes similar to those observed in patients with motor fatigability. There was no correlation between depression and gait attributes. Those patients, who failed to walk for one hour, did not show pathological attractor attributes. Pain during the exercise test can change the gait pattern as well as the attractor attributes. 


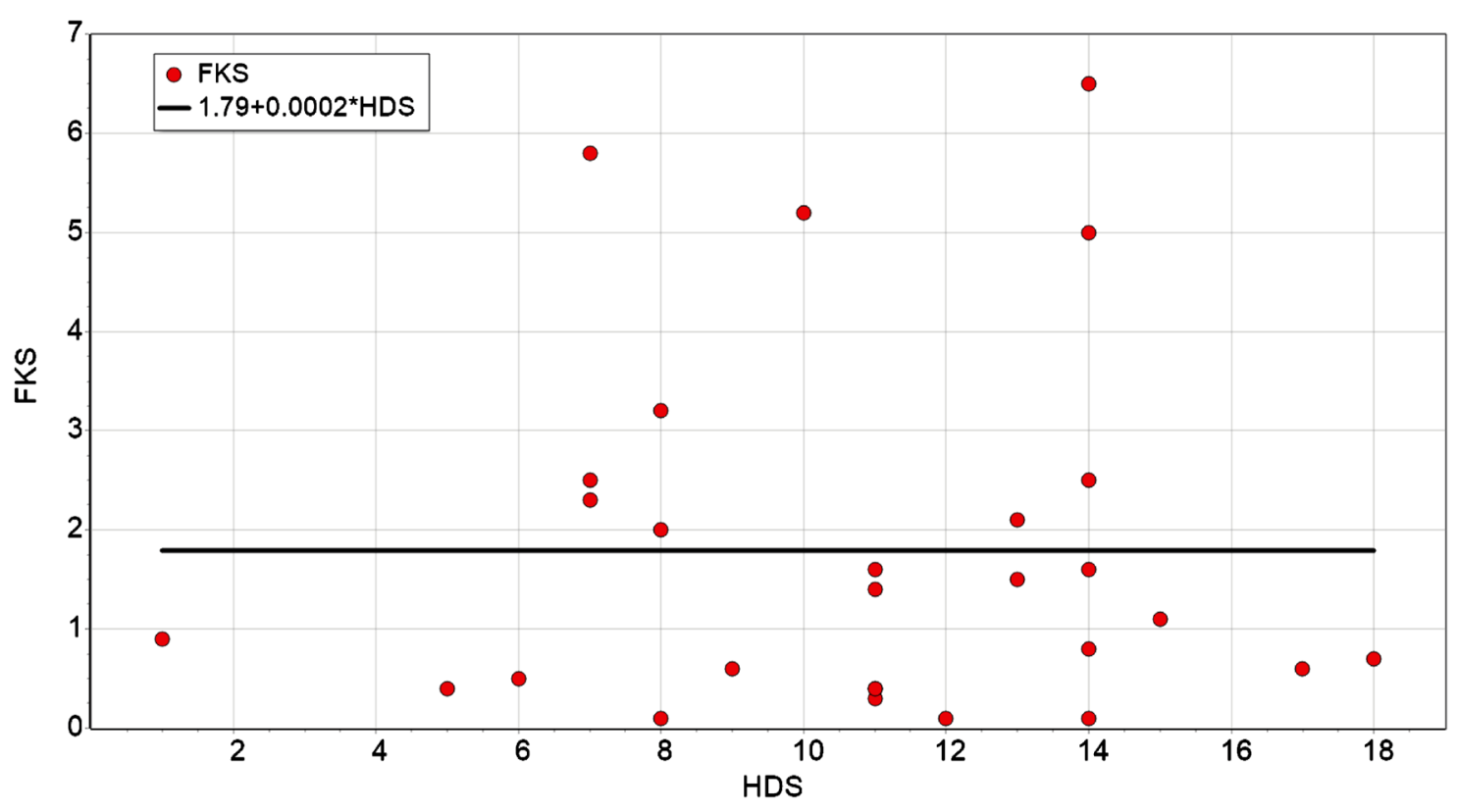

Figure 3. Fatigue Index Kliniken Schmieder (FKS) versus Hamilton Depression Scale (HDS). There is no significant correlation between changes of the Fatigue Index Kliniken Schmieder (FKS) and HDS. The horizontal line being the linear regression function.

Terminology: The proposal to define fatigue as subjective sensation of the patient is very helpful in disentangling different phenomena [8]. Fatigue is rated with questionnaires, while fatigability measures change of performance. The term fatigability, however, has been introduced recently and is beginning to be accepted. Therefore, our publications [11] [12] [14] [15] [16] of the last five years used the term fatigue, whenever fatigability is a better rendering of the patients' condition. Even the Fatigue Index Kliniken Schmieder denotes fatigability.

Since the term fatigability has not been used for a long time, to our knowledge there are not many publications comparing fatigability and fatigue. In our sample of 60 pwMS fatigue as assessed with the FSMC did not correlate with fatigability as determined with the FKS [11]. They are two distinct phenomena.

Another unsettled issue is the clinical relevance of fatigue (the subjective sensation) versus fatigability (the objective decreased performance) as a predictor for early retirement. Although fatigability, which can be measured, might be objective, it is not clear, whether it is more relevant than the subjective sensation of fatigue concerning working capacity.

Patient selection: Patients were not selected right after admittance in the hospital at the peak of their depression, but rather during the phase of their recovery and stabilization. Right after admittance they probably would not have been willing or able to participate. Also clinical administration and the ethical board might have objections to give consent to allow depressive patients to participate in a study during their acute phase. The patients filled in Hamilton Depression scale on the day of their participation indicating moderate depressive symptoms. 
Unfortunately, inclusion criteria were not very rigorous. Exclusion of patients with a second diagnosis especially when involving painful symptoms would have been preferable and might have prevented moderate "false" positive results.

Although participating in a walking group is a standard therapy in patients with depression within this hospital, this inclusion criterion may cause a bias and we cannot necessarily generalize these results to all depressive patients.

The 7-item short form of the HDS is well validated to document the course of depression. A final score $<3$ indicates remission of the depression (McIntyre et al. 2005). The average HDS of our patients was $10.8 \pm 3.8$ indicating moderate depressive symptoms.

Patient performance: Most of the patients (30 out of 32) managed to walk for one hour with an average speed of $4.5 \mathrm{~km} / \mathrm{h}$. This is clearly more than pwMS with motor fatigability who managed to walk on average $810 \mathrm{~m}$ at $1.8 \mathrm{~km} / \mathrm{h}$ [11]. From a practical clinical standpoint this discriminates them from pwMS with motor fatigability. However, our method is velocity normalized to allow comparing attractors of different walking speeds.

Group results of the gait analysis: Group results of the FKS, $\delta M$ and $\delta D$ indicate that the average changes in gait are clearly below those changes in pwMS with motor fatigability (compare Figure 1). The correlational analysis supports the finding, that there is no correlation between depression and attractor attributes (Figure 2).

Individual results: On an individual basis there were six patients with attractor changes in the lower pathological range. Five of them claimed pain during the walking test. This suggests that increasing pain during walking might induce changes in the walking parameters. These, however, are still in the lower range compared to pwMS displaying fatigability. It is remarkable that variability did not increase during the exercise suggesting that $\delta D$ might be more specific for fatigability than $\delta M$.

Again, we would like to stress that this method has been developed not merely to describe group differences between pwMS with and without fatigability, but to diagnose this phenomenon in each individual patient as being present or absent and even to quantify it.

Instrumentation: Sehle et al.'s study [11] investigated pwMS and compared them with healthy volunteers. They used a motion analysis system to determine coordinates and two consecutive numerical derivations to obtain accelerations. In the present study we received accelerations from direct use of the accelerometers. While Sehle et al. used the motion analysis device AS200 (LUKOtronic, Lutz Mechanotroci Technology E.U., Innsbruck, Austria; sampling rate $80 \mathrm{~Hz}$ ) we used accelerometers from Hasomed with a sampling rate of $600 \mathrm{~Hz}$. This might be responsible for minute changes of the readings of variability and attractor values caused by a slightly different marker/sensor mounting. The different sampling rates, however, are not altering the results as we were using the low frequency information of our signals out of a low pass filter (22) with a cutoff at $4.5 \mathrm{~Hz}$.

There are studies in elderly populations assessing the risk of falls or fear of falls by 
analyzing a conventional gait parameter with clinical gait tests [17]. Other studies based on different ways of gait analysis try to characterize the gait pattern in patients with Subcortical Arteriosclerotic Encephalopathy (SAE), Hydrocephalus, Parkinson Disease or other degenerative diseases [18]. Studies use conventional gait parameters to assess the association between depressive symptoms and gait dysfunction [19]. Accelerometers are increasingly used to assess physical activity in pwMS [20]. Assessment of habitual walking behavior by means of accelerometers might also have the potential to investigate the effectiveness of pharmacological interventions [21]. To our knowledge, however, there are no studies similar to ours that have used attractor-based methods to quantify motor fatigability and to delineate fatigability in the presence of depression. This method has the advantage to be highly sensitive to identifying changes in gait pattern and variability during prolonged walking. This makes it a sensitive method to detect motor fatigability particularly in the presence of depression or depressive symptoms. Other causes for attractor and variability changes, however, particularly pain while walking, must be ruled out. This study demonstrates that depression has no significant influence on changes of the FKS. Therefore, FKS is an excellent index describing fatigability even while patients might show severe signs of depression.

\section{Conclusion}

This study shows that changes of gait pattern during depression are not compromising FKS as a reliable indicator for fatigability. Slight changes of attractor attributes can be caused by pain during walking. This can influence results even in the absence of depression. This is important to keep in mind when applying attractor attributes and the measure FKS as diagnostic tools for motor fatigability in pwMS.

\section{Declaration of Conflicting Interests}

The authors declare that there is no conflict of interest.

\section{Acknowledgements}

We appreciate very much the constructive and careful comments of an anonymous reviewer of a previous version of the manuscript.

\section{References}

[1] Freal, J.E., Kraft, G.H. and Coryell, J.K. (1984) Symptomatic Fatigue in Multiple Sclerosis. Archives of Physical Medicine and Rehabilitation, 65, 135-138.

[2] Fisk, J.D., Pontefract, A., Ritvo, P.G., Archibald, C.J. and Murray, T.J. (1994) The Impact of Fatigue on Patients with Multiple Sclerosis. The Canadian Journal of Neurological Sciences (Le journal canadien des sciences neurologiques), 21, 9-14. http://dx.doi.org/10.1017/S0317167100048691

[3] Krupp, L.B. (2003) Fatigue in Multiple Sclerosis: Definition, Pathophysiology and Treatment. CNS Drugs, 17, 225-234. http://dx.doi.org/10.2165/00023210-200317040-00002

[4] Simmons, R.D. (2010) Life Issues in Multiple Sclerosis. Nature Reviews Neurology, 6, 603610. http://dx.doi.org/10.1038/nrneurol.2010.143 
[5] Simmons, R.D., Tribe, K.L. and McDonald, E.A. (2010) Living with Multiple Sclerosis: Longitudinal Changes in Employment and the Importance of Symptom Management. Journal of Neurology, 257, 926-936. http://dx.doi.org/10.1007/s00415-009-5441-7

[6] Penner, I.K., Raselli, C., Stocklin, M., Opwis, K., Kappos, L. and Calabrese, P. (2009) The Fatigue Scale for Motor and Cognitive Functions (FSMC): Validation of a New Instrument to Assess Multiple Sclerosis-Related Fatigue. Multiple Sclerosis, 15, 1509-1517. http://dx.doi.org/10.1177/1352458509348519

[7] Veauthier, C. and Paul, F. (2014) Sleep Disorders in Multiple Sclerosis and Their Relationship to Fatigue. Sleep Medicine, 15, 5-14.

http://dx.doi.org/10.1016/j.sleep.2013.08.791

[8] Kluger, B.M., Krupp, L.B. and Enoka, R.M. (2013) Fatigue and Fatigability in Neurologic Illnesses: Proposal for a Unified Taxonomy. Neurology, 80, 409-416.

http://dx.doi.org/10.1212/WNL.0b013e31827f07be

[9] Claros-Salinas, D., Bratzke, D., Greitemann, G., Nickisch, N., Ochs, L. and Schroter, H. (2010) Fatigue-Related Diurnal Variations of Cognitive Performance in Multiple Sclerosis and Stroke Patients. Journal of the Neurological Sciences, 295, 75-81.

http://dx.doi.org/10.1016/j.jns.2010.04.018

[10] Neumann, M., Sterr, A., Claros-Salinas, D., Gutler, R., Ulrich, R. and Dettmers, C. (2014) Modulation of Alertness by Sustained Cognitive Demand in MS as Surrogate Measure of Fatigue and Fatigability. Journal of the Neurological Sciences, 340, 178-182. http://dx.doi.org/10.1016/j.jns.2014.03.024

[11] Sehle, A., Vieten, M., Sailer, S., Mundermann, A. and Dettmers, C. (2014) Objective Assessment of Motor Fatigue in Multiple Sclerosis: The Fatigue Index Kliniken Schmieder (FKS). Journal of Neurology, 261, 1752-1762. http://dx.doi.org/10.1007/s00415-014-7415-7

[12] Vieten, M.M., Sehle, A. and Jensen, R.L. (2013) A Novel Approach to Quantify Time Series Differences of Gait Data Using Attractor Attributes. PLOS ONE, 8, e71824. http://dx.doi.org/10.1371/journal.pone.0071824

[13] McIntyre, R.S., Konarski, J.Z., Mancini, D.A., Fulton, K.A., Parikh, S.V., Grigoriadis, S., et al. (2005) Measuring the Severity of Depression and Remission in Primary Care: Validation of the HAMD-7 Scale. Canadian Medical Association Journal, 173, 1327-1334. http://dx.doi.org/10.1503/cmaj.050786

[14] Sehle, A., Vieten, M., Mundermann, A. and Dettmers, C. (2014) Difference in Motor Fatigue between Patients with Stroke and Patients with Multiple Sclerosis: A Pilot Study. Frontiers in Neurology, 5, 279. http://dx.doi.org/10.3389/fneur.2014.00279

[15] Sehle, A., Mundermann, A., Starrost, K., Sailer, S., Becher, I., Dettmers, C., et al. (2011) Objective Assessment of Motor Fatigue in Multiple Sclerosis Using Kinematic Gait Analysis: A Pilot Study. Journal of Neuroengineering and Rehabilitation, 8, 59. http://dx.doi.org/10.1186/1743-0003-8-59

[16] Dettmers, C., Sulzmann, M., Ruchay-Plossl, A., Gutler, R. and Vieten, M. (2009) Endurance Exercise Improves Walking Distance in MS Patients with Fatigue. Acta Neurologica Scandinavica, 120, 251-257. http://dx.doi.org/10.1111/j.1600-0404.2008.01152.x

[17] Moreira Bde, S., Dos Anjos, D.M., Pereira, D.S., Sampaio, R.F., Pereira, L.S., Dias, R.C., et al. (2016) The Geriatric Depression Scale and the Timed up and Go Test Predict Fear of Falling in Community-Dwelling Elderly Women with Type 2 Diabetes Mellitus: A CrossSectional Study. BMC Geriatrics, 16, 56. http://dx.doi.org/10.1186/s12877-016-0234-1

[18] Gor-Garcia-Fogeda, M.D., Cano de la Cuerda, R., Carratala Tejada, M., Alguacil-Diego, I.M. and Molina-Rueda, F. (2016) Observational Gait Assessments in People with Neuro- 
logical Disorders: A Systematic Review. Archives of Physical Medicine and Rehabilitation, 97, 131-140. http://dx.doi.org/10.1016/j.apmr.2015.07.018

[19] Brandler, T.C., Wang, C., Oh-Park, M., Holtzer, R. and Verghese, J. (2012) Depressive Symptoms and Gait Dysfunction in the Elderly. American Journal of Geriatric Psychiatry, 20, 425-432. http://dx.doi.org/10.1097/JGP.0b013e31821181c6

[20] Gijbels, D., Alders, G., Van Hoof, E., Charlier, C., Roelants, M., Broekmans, T., et al. (2010) Predicting Habitual Walking Performance in Multiple Sclerosis: Relevance of Capacity and Self-Report Measures. Multiple Sclerosis, 16, 618-626.

http://dx.doi.org/10.1177/1352458510361357

[21] Stellmann, J.P., Neuhaus, A., Gotze, N., Briken, S., Lederer, C., Schimpl, M., et al. (2015) Ecological Validity of Walking Capacity Tests in Multiple Sclerosis. PLoS ONE, 10, e0123822. http://dx.doi.org/10.1371/journal.pone.0123822

\section{Abbreviations}

HDS: Hamilton Depression Scale; MS: Multiple Sclerosis; pwMS: persons with MS; SAE: Subcortical Arteriosclerotic Encephalopathy; The changes between two measurements: $\delta M$ difference of the attractors $-\delta D$ difference of the variability $-\delta F=\mathrm{FKS}$ Fatigue Index Kliniken Schmieder.

\section{Submit or recommend next manuscript to SCIRP and we will provide best service for you:}

Accepting pre-submission inquiries through Email, Facebook, LinkedIn, Twitter, etc. A wide selection of journals (inclusive of 9 subjects, more than 200 journals)

Providing 24-hour high-quality service

User-friendly online submission system

Fair and swift peer-review system

Efficient typesetting and proofreading procedure

Display of the result of downloads and visits, as well as the number of cited articles

Maximum dissemination of your research work

Submit your manuscript at: http://papersubmission.scirp.org/

Or contact health@scirp.org 